PROCEEDINGS OF THE

AMERICAN MATHEMATICAL SOCIETY

Volume 134, Number 7, Pages 2091-2097

S 0002-9939(06)08201-3

Article electronically published on January 6, 2006

\title{
THE GENERALIZED LICHNEROWICZ PROBLEM: UNIFORMLY QUASIREGULAR MAPPINGS AND SPACE FORMS
}

\author{
GAVEN MARTIN, VOLKER MAYER, AND KIRSI PELTONEN
}

(Communicated by Juha M. Heinonen)

\begin{abstract}
A uqr mapping of an $n$-manifold $M$ is a mapping which is rational with respect to a bounded measurable conformal structure on $M$. Remarkably, the only closed manifolds on which locally (but not globally) injective uqr mappings act are Euclidean space forms. We further characterize space forms admitting uniformly quasiregular self mappings and we show that the space forms admitting branched uqr maps are precisely the spherical space forms. We further show that every non-injective uqr map of a Euclidean space form is a quasiconformal conjugate of a conformal map. This is not true if the non-injective hypothesis is removed.
\end{abstract}

\section{INTRODUCTION}

A quasiregular map $f$ which admits uniform control of the distortion of all its iterates is called uniformly quasiregular, abbreviated uqr. Such maps are always conformal (or rational) with respect to some bounded measurable Riemannian structure. Here we will always suppose uqr mappings to be non-injective (otherwise we call the map quasiconformal). Because of Rickman's version of Montel's theorem Ri] the dynamical system associated with the iterates of a non-injective uqr mapping of the $n$-sphere closely parallel (in particular with regard to the Fatou-Julia theory) that of the iteration of rational maps in the plane [IM1, M1, M2, HMM] with a few subtle differences connected with rigidity phenomena [MM], one of which underpins our main result.

Here we shall consider such mappings acting on closed manifolds $M$ of dimension at least two and our problem is to determine what kind of manifolds admit the action of such a map and also to determine what kind of uqr mappings can act on a given manifold. A result of Sullivan (see Tukia-Väisälä [TV]) shows that any topological $n$-manifold, $n \neq 4$, admits a quasiconformal structure and this is enough to define a bounded measurable Riemannian structure and what it means to be quasiregular or uqr, Ri]. In four dimensions we shall have to suppose our manifolds admit such a structure.

Received by the editors August 28, 2003 and, in revised form, February 17, 2005

2000 Mathematics Subject Classification. Primary 30D05; Secondary 32H02.

Key words and phrases. Uniformly quasiregular mappings, space forms.

This research was supported in part by the Marsden Fund (NZ) and The Royal Society of NZ (James Cook Fellowship). 
Note that a uqr map $f: M \rightarrow M$ is surjective since the continuity and openness of a quasiregular map [Ri] implies that the image $f M$ is both compact and open; hence $f M=M$.

The first part of our problem is a non-injective version of the answer given by Ferrand $[\mathrm{F}$ to a conjecture of Lichnerowicz $[\mathrm{L}$. She showed that, up to quasiconformal equivalence, the only compact manifold which admits a non-compact quasiconformal group action is the standard $n$-sphere $\mathbb{S}^{n}$. If there is a uqr map $f$ of a closed manifold $M$, then the semi-group $\left\{f^{n}\right\}_{n=1}^{\infty}$ is non-compact (in fact the Julia set of $f$ is always non-empty). Therefore, the existence of such a map should imply severe restrictions on the manifold $M$. The first of these is the following obstruction for the existence of uqr maps:

Theorem 1.1. If $M^{n}$ is a closed n-manifold and $f: M^{n} \rightarrow M^{n}$ a non-injective uqr mapping, then there exists a non-constant quasiregular mapping $g: \mathbb{R}^{n} \rightarrow M^{n}$.

Manifolds admitting such an $f$ are called $q r$-elliptic, and answering a question of Gromov, Varopoulos, Saloff-Coste and Coulhon [VSC] showed that $M$ must have a fundamental group of at most polynomial growth.

Our contribution to the generalized Lichnerowicz problem of determining all closed manifolds which admit non-injective uqr mappings is the following:

Theorem 1.2. Let $f$ be a non-injective uqr map of the closed manifold $M$ and suppose that $f$ is locally homeomorphic, that is, the branch set $B_{f}=\emptyset$. Then $M$ is the quasiconformal image of a Euclidean space form.

By a Euclidean space form we mean the quotient of $\mathbb{R}^{n}$ under a Bieberbach group (co-finite volume lattice) $\Gamma \subset \operatorname{Isom}\left(\mathbb{R}^{n}\right)$. The two other types of space forms are the quotients by torsion free co-finite volume lattices of isometries of the $n$-sphere and of hyperbolic $n$-space.

Theorem 1.5 below further asserts that after the quasiconformal change of coordinates suggested by Theorem 1.2, the map $f$ can be made conformal. However we first state

Theorem 1.3. If $M$ is quasiconformally equivalent to a euclidean space form, then $M$ admits no branched quasiregular (and in particular no branched uqr) mappings.

Remark 1.4. The proof we give for Theorem 1.3 requires only the topological properties of quasiregular mappings. Consequently, the previous result can be generalized as follows:

Suppose $M$ is homeomorphic to a Euclidean space form. Then $M$ does not admit an orientation preserving, discrete and open map which is branched.

In the case of the sphere, lens spaces and other spherical manifolds the existence of uqr maps is due to [M1 and [P. These results suggest that there are few uqr mappings in three or more dimensions compared with the space of rational functions of $\mathbb{S}^{2}$. For Euclidean space forms we can now give a complete description of the possible uqr mappings:

Theorem 1.5. Any non-injective uqr map of a closed Euclidean space form $M$ is the quasiconformal conjugate of a conformal map.

We remark that, in this second result, we no longer suppose that the map has to be locally injective. Theorem 1.5 is a surprising fact because it is false for globally 
injective mappings. Indeed, it turns out that there are uniformly quasiconformal (even bi-Lipschitz) maps of three (or higher) dimensional tori which cannot be quasiconformally conjugate to a conformal map [M1].

Finally, we can distinguish space forms according to the type of uqr maps they support:

Theorem 1.6. If $M$ is a closed space form, then we have the following characterization:

(1) $M$ admits a branched uqr map if and only if $M$ is a spherical space form.

(2) $M$ admits a non-injective, locally injective uqr map if and only if $M$ is a Euclidean space form.

(3) $M$ admits no non-injective uqr map if and only if $M$ is a hyperbolic space form.

We suggest that the correct counterpart for the Lichnerowicz conjecture for uqr maps could be that if a closed manifold supports a non-injective uqr map, then it must be a finite product $M_{1} \times \cdots \times M_{k}$ of closed manifolds $M_{i}$ which are quasiconformal images of either Euclidean or spherical space forms. It remains an interesting open question whether for example a space like $\mathbb{S}^{2} \times \mathbb{S}^{2} \# \mathbb{S}^{2} \times \mathbb{S}^{2}$ supports uqr maps. This manifold was very recently proven to be elliptic [Ri] and perhaps represents a best candidate as a counterexample to the conjecture.

\section{Proofs}

Proof of Theorem [1.1. Suppose $f$ is a non-injective uqr mapping acting on a closed manifold $M$. We may asume that the Julia set is not empty and contains a point $x_{0}$. Introduce coordinates $\left(U_{x_{0}}, h\right)$, where $U_{x_{0}}$ is an open set in $M$ containing $x_{0}$ and $h: U_{x_{0}} \rightarrow \mathbb{B}^{n}$ is a 2-bi-Lipschitz map. We get a non-normal family $\mathcal{F}=\left\{\hat{f}_{k}\right\}$ of mappings $\hat{f}_{k}=f^{k} \circ h^{-1}: \mathbb{B}^{n} \rightarrow M$. Hence there exist positive numbers $\rho_{j} \searrow 0$ and points $x_{j} \rightarrow 0, x_{j} \in \mathbb{B}^{n}$ and mappings $\hat{f}_{j} \in \mathcal{F}$ so that $\hat{f}_{j}\left(x_{j}+\rho_{j} x\right) \rightarrow \psi(x)$ locally uniformly on $\mathbb{R}^{n}$, where $\psi: \mathbb{R}^{n} \rightarrow M$ is a non-constant quasiregular map. See [IM2, Theorems 19.7.3, 19.9.3].

Recall that the conical set of a uqr map is, using the notation of the above proof, the set of those points $x_{0}$ in the Julia set for which the ratio $\left|x_{j}-x_{0}\right| / \rho_{j}$ stays bounded with a uniform constant when $j \rightarrow \infty$.

Proof of Theorem 1.2. First, some general remarks. Suppose $f: M \rightarrow M$ is a non-injective uqr map with empty branch set. Since $M$ is compact we may find a point $x_{0}$ in the Julia set $\mathcal{J}_{f}$ of $f$. It is now possible, as in the proof of Theorem 1.1 to construct a family $\mathcal{F}=\{\hat{f}\}$ of mappings $\hat{f}: \mathbb{B}^{n} \rightarrow M$ and find positive numbers $\rho_{j} \searrow 0$ and points $x_{j} \rightarrow 0, x_{j} \in \mathbb{B}^{n}$ and mappings $\hat{f}_{j} \in \mathcal{F}$ so that $\hat{f}_{j}\left(x_{j}+\rho_{j} x\right) \rightarrow \psi(x)$ locally uniformly in $\mathbb{R}^{n}$, where $\psi: \mathbb{R}^{n} \rightarrow M$ is a non-constant quasiregular map. Now clearly the limit map $\psi$ is also a local homeomorphism. Due to the Picard-type theorem of [HR] $\psi$ omits at most $q<\infty$ points (the number $q$ depends only on the dimension $n$ and the dilatation of the map $\psi$ ). Denote by $\tilde{\psi}: \mathbb{R}^{n} \rightarrow \tilde{M}$ the lift of $\psi$ under a locally isometric covering map $\pi: \tilde{M} \rightarrow M$, where $\tilde{M}$ is the covering space of $M$. If the fundamental group of $M$ is infinite the mapping $\psi$ is necessarily a surjection, since otherwise the lifted map $\tilde{\psi}$ would omit infinitely many points (corresponding to every omitted value of $\psi$ ) and this is again 
impossible due to $[\mathrm{HR}$. The covering space $\tilde{M}$ is hence a homeomorphic image of Euclidean $n$-space $\mathbb{R}^{n}$. This homeomorphism is clearly quasiconformal.

If the fundamental group of $M$ is finite, then the universal cover is closed and simply connected, so the lift of $f$ will be a locally homeomorphic mapping, which the monodromy theorem tells us will be a homeomorphism. This lift $\hat{f}$ will be uniformly quasiconformal and not periodic (as is easily deduced from the relation $f^{m} \circ \pi=\pi \circ \hat{f}$ and the non-injectivity of $f$ ). As $\langle\hat{f}\rangle$ is non-compact, Ferrand's theorem [F] shows this universal cover to be the $n$-sphere. But then $f^{m} \circ \pi: \mathbb{S}^{n} \rightarrow M$ is also a universal covering (necessarily of the same degree as $\pi$ ), so $f$ is a homeomorphism contrary to supposition.

We now show that $M$ is a Euclidean space form. Suppose $f: M \rightarrow M$ is a non-injective uqr map with empty branch set. We first show that every point of the Julia set is a conical point. Thus let $x_{0}$ be a point of the Julia set $\mathcal{J}_{f}$ of $f$. It is the local injectivity condition of $f$ which will ensure that $x_{0}$ is conical. To see this denote $x_{n}=f^{n}\left(x_{0}\right)$ and let $n_{j} \rightarrow \infty$ such that the limit $y=\lim _{j \rightarrow \infty} x_{n_{j}}$ exists. Set $B=\mathbb{B}(y, r) \subset M$. If

$$
r_{j}=\sup \left\{r>0 ; f^{n_{j}}\left(B\left(x_{0}, r\right)\right) \subset B\right\},
$$

then, with the notation of the proof of Theorem 1.1, $\Psi_{j}(x)=\hat{f}_{n_{j}}\left(r_{j} x\right)$ defines on the unit ball $\mathbb{B}^{n}$ a normal family of uniformly quasiconformal mappings (note that all the inverse branches of $f^{n_{j}}$ are defined on $B$ ). We therefore may suppose that the limit $\Psi=\lim _{j \rightarrow \infty} \Psi_{j}$ exists. From the distortion properties of quasiconformal mappings and the choice of $r_{j}$ we see that the limit map $\Psi$ is non-constant and hence quasiconformal from $\mathbb{B}^{n}$ onto say $\Omega \subset B$. It follows that the conical set coincides with the Julia set.

We now make the following additional observation to show that every point of $M$ is in the Julia set. If $j_{0}>>1$ such that the distance $\left\|x_{n_{j}}-y\right\|<r / 100$ and if $j>j_{0}$ such that

$$
U=\hat{f}_{n_{j_{0}}}\left(\mathbb{B}\left(0, r_{j}\right)\right) \subset \mathbb{B}\left(x_{n_{j_{0}}}, \frac{r}{1000 K}\right),
$$

then $f^{n_{j}-n_{j_{0}}}(U) \supset \bar{U}$. The map $g=f^{n_{j}-n_{j_{0}}}: U \rightarrow g(U) \subset B$ being quasiconformal, must have a repelling fixed point $p$. Replacing $f$ by an iterate, if necessary, we may suppose that $f$ has a repelling fixed point. Thus we can choose a lift $F$ of $f$ to the universal cover $\mathbb{R}^{n}$ that will also have a repelling fixed point. The iterates of $F$ are not equicontinuous near any point $x$ of $\mathbb{R}^{n}$. Indeed it is shown in GM that in this case $F$ is quasiconformally conjugate to the map $x \mapsto 2 x$, whose iterates clearly do not form an equicontinuous family. Thus $f$ is nowhere equicontinuous and $\mathcal{J}_{f}=M$ is purely conical by what we have proved above.

Now, any equivariant measurable conformal structure preserved by the uqr mapping $f$ is continuous in measure at a conical point. And we know there is at least one such structure. We can hence apply the rigidity theorem for uqr maps [MM, Theorem 6.1 ] to deduce that $f$ is actually a Lattès type map. Especially $F$ is then an expanding similarity and $M$ is a Euclidean space form.

Proof of Theorem 1.3. Suppose $M=\mathbb{R}^{n} / \Gamma$ is a Euclidean space form and $f: M \rightarrow$ $M$ a non-constant quasiregular mapping. First of all, we may suppose that $\Gamma$ consists of translations only and, because of compactness of $M$, that $\Gamma$ is isomorphic to $\mathbb{Z}^{n}$. This is because of Bieberbach's theorem (see $[\mathrm{W}]$ ): the translation subgroup $\mathcal{T}$ of $\Gamma$ is a normal subgroup of finite index and $\mathbb{R}^{n} / \mathcal{T}$ is a normal covering space of 
$M$. Therefore it suffices to consider $\mathbb{R}^{n} / \mathcal{T}$ together with a lift of $f$ to this covering of $M$ instead of $M, f$.

Denote by $F: \mathbb{R}^{n} \rightarrow \mathbb{R}^{n}$ a quasiregular lift of $f$ and let $D$ be a fundamental domain of $M$ containing the origin. We can further assume that $F(0) \in D$. Then there exist a group homomorphism $A: \Gamma \rightarrow \Gamma$ so that for any $x \in \mathbb{R}^{n}$ and $\gamma(x)=$ $x+v, \gamma \in \Gamma$, the following holds:

$$
F(x+v)=F(x)+A(v) .
$$

Since $\Gamma$ is a translation group isomorphic to $\mathbb{Z}^{n}$, the above homomorphism is given by a linear map $A: \mathbb{R}^{n} \rightarrow \mathbb{R}^{n}$. Furthermore, $A$ is invertible because $A \Gamma$ is again isomorphic to $\mathbb{Z}^{n}$.

We now show that $F$ has an invertible derivative at $\infty$, which concludes the proof. Indeed, we then have $i(\infty, F)=1$. On the other hand, this local index does agree with the degree of the map $F$ since the only preimage of $\infty$ is infinity itself. In other words, $F$ is a homeomorphism.

Let $i(x)=\frac{x}{\|x\|^{2}}$ be the chart at infinity, denote $y=i(x)$,

$$
G=i \circ F \circ i \text { and } A^{*}=i \circ A \circ i \text {. }
$$

Clearly $G(0)=0$. For $x$ close to the origin,

$$
G(x)=\frac{F(y)}{\|F(y)\|^{2}}=\frac{F\left(y_{0}\right)+A\left(y-y_{0}\right)}{\left\|F\left(y_{0}\right)+A\left(y-y_{0}\right)\right\|^{2}}=\frac{A(y)+\left(F\left(y_{0}\right)-A\left(y_{0}\right)\right)}{\left\|A(y)+\left(F\left(y_{0}\right)-A\left(y_{0}\right)\right)\right\|^{2}}
$$

where $y_{0} \in D$ such that there is $\gamma \in \Gamma$ with $\gamma\left(y_{0}\right)=y_{0}+v=y$. Clearly

$$
B\left(y_{0}\right)=F\left(y_{0}\right)-A\left(y_{0}\right)
$$

is bounded on $D$. Therefore,

$$
\frac{\|A(y)\|^{2}}{\left\|A(y)+B\left(y_{0}\right)\right\|^{2}} \leq \frac{1}{1-\left(\frac{\left\|B\left(y_{0}\right)\right\|}{\|A(y)\|}\right)^{2}} \leq 1+C \frac{1}{\|A(y)\|^{2}} \leq 1+C^{\prime}\|x\|^{2} .
$$

Similarly

This leads to

$$
\frac{\|A(y)\|^{2}}{\left\|A(y)+B\left(y_{0}\right)\right\|^{2}} \geq 1-C^{\prime \prime}\|x\|^{2}
$$

$$
\begin{aligned}
G(x) & =\frac{A(y)}{\|A(y)\|^{2}} \frac{\|A(y)\|^{2}}{\left\|A(y)+B\left(y_{0}\right)\right\|^{2}}+\frac{B\left(y_{0}\right)}{\left\|A(y)+B\left(y_{0}\right)\right\|^{2}} \\
& =A^{*}(x)\left(1+O\left(\|x\|^{2}\right)\right)+O\left(\|x\|^{2}\right) \\
& =A^{*}(x)+O\left(\|x\|^{2}\right),
\end{aligned}
$$

meaning that $D G(0)=A^{*}$.

Proof of Theorem 1.5. Theorem 1.3 gives the local injectivity of the map. We note that alternatively this can be seen shortly by using more dynamical arguments as follows. Let $M=\mathbb{R}^{n} / \Gamma$ be a Euclidean space form and $f$ a uqr map of $M$. Consider $\pi: \mathbb{R}^{n} \rightarrow M$ the natural projection and take $F$ (also uqr) a lift of $f$. We proceed by contradiction and suppose that $F$ is not injective.

From the proof of Theorem 1.2 the map $F$ extends at infinity to a uqr map of the sphere $\overline{\mathbb{R}}^{n}$ and $\infty$ is a completely invariant fixed point which is super-attracting since we supposed $F$ is not quasiconformal; indeed since $\infty$ is completely invariant, the local index of $F$ at that point is equal to the degree of $F$ and hence $\infty$ is in the 
branch set of the extended map. It then follows that the Fatou set of $F$ contains an open neighborhood of $\infty$, the basin of attraction of $\infty$. See [HMM] for details. From standard estimates near $\infty$ we have, after replacing $\mathrm{F}$ by a power of itself if necessary, the following: there is $P>1$ and $R>0$ such that

$$
\|F(x)\| \geq\|x\|^{P} \quad \text { provided } \quad\|x\|>R .
$$

Now, for $\gamma \in \Gamma$ there is $\gamma^{\prime} \in \Gamma$ such that $F \circ \gamma^{N}=\left(\gamma^{\prime}\right)^{N} \circ F$. There are $U, V \in$ $O(n)$ and $v, w \in \mathbb{R}^{n}$ such that $\gamma^{N}(x)=U^{N} x+N v$ and $\left(\gamma^{\prime}\right)^{N}(x)=V^{N} x+N w$. We take $\gamma$ parabolic such that $\left\|\gamma^{N}(0)\right\|>R$ for all $N$ large enough. Then:

$$
\left\|F \circ \gamma^{N}(0)\right\| \geq\left\|\gamma^{N}(0)\right\|^{P}=(N\|v\|)^{P}
$$

and thus

$$
(N\|v\|)^{P} \leq\left\|\left(\gamma^{\prime}\right)^{N}(F(0))\right\| \leq\|F(0)\|+N\|w\| .
$$

This is impossible if $N$ has been chosen large enough. The map $F$ is in fact quasiconformal.

To conclude the proof we will look for a quasiconformal map $\Phi: \mathbb{R}^{n} \rightarrow \mathbb{R}^{n}$, a Bieberbach group $\Gamma^{\prime}$ and a conformal (loxodromic) map $G: \mathbb{R}^{n} \rightarrow \mathbb{R}^{n}$ such that

$$
\Phi:\langle F, \Gamma\rangle \rightarrow\left\langle G, \Gamma^{\prime}\right\rangle
$$

meaning that $\Phi$ conjugates the maps $F, G$ and the groups $\Gamma, \Gamma^{\prime}$. It suffices then to project $\Phi$ to a quasiconformal map $\varphi: M \rightarrow M^{\prime}=\mathbb{R}^{n} / \Gamma^{\prime}$ because this new map conjugates $f$ and $g: M^{\prime} \rightarrow M^{\prime}$, the projection of $G$.

In order to do this we take a bounded measurable conformal structure $\mu$ on $M$ for which $f$ is conformal. Lift this structure to $\mathbb{R}^{n}$ to a structure for which the group $\langle F, \Gamma\rangle$ is conformal and again use a renormalization to find $\Phi$.

Proof of Theorem 1.6. The existence of a branched uqr map for an arbitrary spherical space form $M^{n}$ is proved in $\mathrm{P}$. There one starts with an arbitrary branched covering map $f: M^{n} \rightarrow \mathbb{S}^{n}$ followed by the covering projection $g: \mathbb{S}^{n} \rightarrow M^{n}$. The mapping $g \circ f: M^{n} \rightarrow M^{n}$ can now be modified to a uqr mapping by the so-called trapping method. The branch set does not change under the construction.

For a hyperbolic space form there are no non-injective uqr maps at all. This follows from Theorem 1.1, since hyperbolicity implies exponential volume growth for the fundamental group. Fundamental groups of manifolds quasiregularly covered by $\mathbb{R}^{n}$ have at most polynomial growth of order $n$ VSC].

The non-existence of a branched uqr map in the Euclidean space form case is Theorem 1.3 above. The existence of a non-injective uqr map can be shown by constructing Lattès type maps. This can be seen by using the following characterization [R, Theorem 13, p. 373]. Suppose $\Gamma$ is the fundamental group of a Euclidean space form $M$. The group $\Gamma$ consists of the translation subgroup and the rotation subgroup. One can introduce coordinates $\left\{e_{i}\right\}_{i=1}^{n}$ of the covering space $\mathbb{R}^{n}$ in such a way that the elements of the translation part are of the form $x \mapsto x+\sum_{i=1}^{n} m_{i} e_{i}$, $m_{i} \in \mathbb{Z}$ and the general element is of the form

$$
x \mapsto R(x)+\sum_{i=1}^{n} \frac{n_{i}}{g} e_{i},
$$

where the matrix of $R$ representing the rotational part, when presented in this fixed base, contains integer entries only, $n_{i} \in \mathbb{Z}$ and $g$ is the order of the rotational part. 
The Lattès type map $f: M \rightarrow M$ now arises as a solution of the equation

$$
(f \circ \pi)(x)=(\pi \circ A)(x),
$$

where $\pi: \mathbb{R}^{n} \rightarrow M$ is the conformal covering projection. For the dilation map $A: \mathbb{R}^{n} \rightarrow \mathbb{R}^{n}$ one can choose $A: x \mapsto 2 g x$, which preserves the lattice identifications as needed. See [M2] for details on Lattès type map constructions.

\section{REFERENCES}

[F] J. Ferrand, Transformations conformes et quasi-conformes des variétés riemanniennes compactes (démonstration de la conjecture de A. Lichnerowicz), Acad. Roy. Belg. Cl. Sci. Mém. Coll. in-8deg(2), 39, 44 pp. (1971). MR0322739 (48:1100)

[GM] F. Gehring and G. Martin, Discrete quasiconformal groups I, Proc. London Math. Soc. (3), 55, (1987), 331-358. MR0896224 (88m:30057)

[HMM] A. Hinkkanen, G. Martin and V. Mayer, Local dynamics of uniformly quasiregular mappings, Math. Scand., 95, (2004), 80-100. MR2091483 (2005f:37094)

[HR] I. Holopainen and S. Rickman, Ricci curvature, Harnack functions and Picard type theorems for quasiregular maps, Analysis and Topology, World Sci. Publ., (1998), 315-326. MR.1667818(99j:30026)

[IM1] T. Iwaniec and G. Martin, Quasiregular semigroups, Ann. Acad. Sci. Fenn. Ser. AI Math., 21, (1996), 241-254. MR1404085 (97i:30032)

[IM2] T. Iwaniec and G. Martin, Geometric function theory and non-linear analysis, Oxford Mathematical Monographs, 2001. MR.1859913 (2003c:30001)

[L] A. Lichnerowicz, Sur les transformations conformes d'une variété riemannienne compacte. (Italian) C. R. Acad. Sci. Paris, 259, (1964), 697-700. MR0166734 (29:4007)

[MM] G. Martin and V. Mayer, Rigidity in holomorphic and quasiregular dynamics, Trans. Amer. Marth. Soc. 355, (2003), 4349-4363. MR1990755 (2004i:37095)

[M1] V. Mayer, Cyclic parabolic quasiconformal groups that are not the quasicoformal conjugates of Möbius groups, Ann. Acad. Sci. Fenn. Ser. AI Math., 18, (1993), 147-154. MR.1207901 (95f:30032)

[M2] V. Mayer, Uniformly quasiregular mappings of Lattès type, Conformal Geometry and Dynamics, 1, (1997), 104-111. MR1482944 (98j:30017)

[P] K. Peltonen, Examples of UQR mappings, Conformal Geometry and Dynamics, 3, (1999), 158-163. MR1718708 (2001i:30017)

[Ri] S. Rickman, Simply connected quasiregularly elliptic 4-manifolds, preprint.

[R] W. Rinow, Die innere Geometrie der Metrischen Räume, Springer, 1961. MR0123969 (23:A1290)

[TV] P. Tukia and J. Väisälä, Lipschitz and quasiconformal approximation and extension., Ann. Acad. Sci. Fenn. Ser. A I Math., 6, (1981), 303-342 (1982) MR0658932 (84a:57016)

[VSC] N.Th. Varopoulos, L. Saloff-Coste and T.Coulhon, Analysis and geometry on groups, Cambridge Univ. Press, Cambridge, 1992. MR.1218884 (95f:43008)

[W] J.A. Wolf, Spaces of constant curvature, McGraw-Hill Series in Higher Mathematics (1967). MR0217740 (36:829)

Institute of Information and Mathematical Sciences, Massey University, Auckland, New ZeALAND

E-mail address: g.j.martin@massey.ac.nz

UFR de Mathématiques, UMR 8524 du CNRS, Université de Lille I, 59655 Villeneuve D'Ascq Cedex, France

E-mail address: volker.mayer@univ-lille1.fr

Helsinki University of Technology, P.O. Box 1100, Fin-02015 Espoo, Finland

E-mail address: kirsi.peltonen@helsinki.fi 